\title{
Tratamiento de reposición hidroelectrolítica en el paciente deshidratado por cólera.
}

\section{Hydroelectrolyte treatment in dehydration by acute cholera diarrhea.}

\section{Cieza Z Javier*, Estremadoyro Luis**}

*Servicio de Nefrología, Hospital Nacional Cayetano Heredia. Profesor Asociado, Departamento de Medicina. Universidad Peruana Cayetano Heredia. Lima, Perú.

**Servicio de Nefrología, Hospital Nacional Cayetano Heredia. Profesor Auxiliar, Departamento de Medicina. Universidad Peruana Cayetano Heredia. Lima, Perú.

Rev Med Hered 1995;6(3): 154-158.

Un adecuado enfoque del tratamiento está sustentado en la demanda de pacientes, en la infraestructura de salud existente y en los recursos humanos y económicos dispuestos a los servicios.

La experiencia en el Perú y las bajas tasas de letalidad registradas durante los tres primeros años del cólera, no significan una homogeneidad de los resultados. En las zonas urbanas, la letalidad por el cólera ha sido mucho más baja que en la zonas rurales (1).

Dentro de la ciudad de Lima, las tasas de letalidad han variado entre los diferentes hospitales y dentro de estos, han variado en el tiempo ( Figura $\mathrm{N}^{\mathrm{o}} 1$ ).

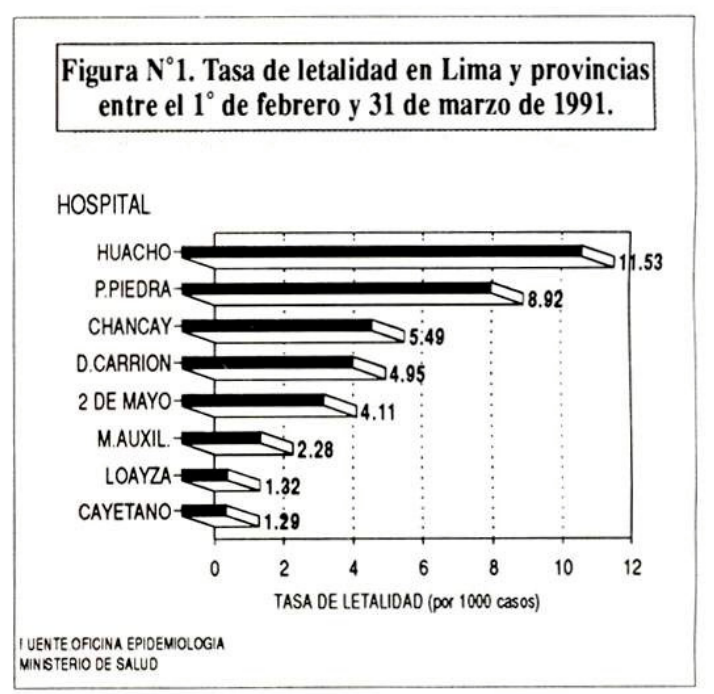


Para una comunidad afectada por la enfermedad, la proximidad de los servicios de salud es un punto crítico para disminuir las tasas de morbilidad y letalidad. Esto queda reflejado en las diferentes tasas de letalidad entre la población urbana y rural.

Otra observación importante es el efecto sobre la letalidad de la experiencia a través del tiempo en los diferentes hospitales de Lima (Figura No2). El Hospital Cayetano Heredia ubicado en el cono norte de la ciudad de Lima, ha tenido una de las tasas más bajas de letalidad dentro de los hospitales del Perú. La organización de la institución en función de una gran demanda resultó relevante en los resultados.

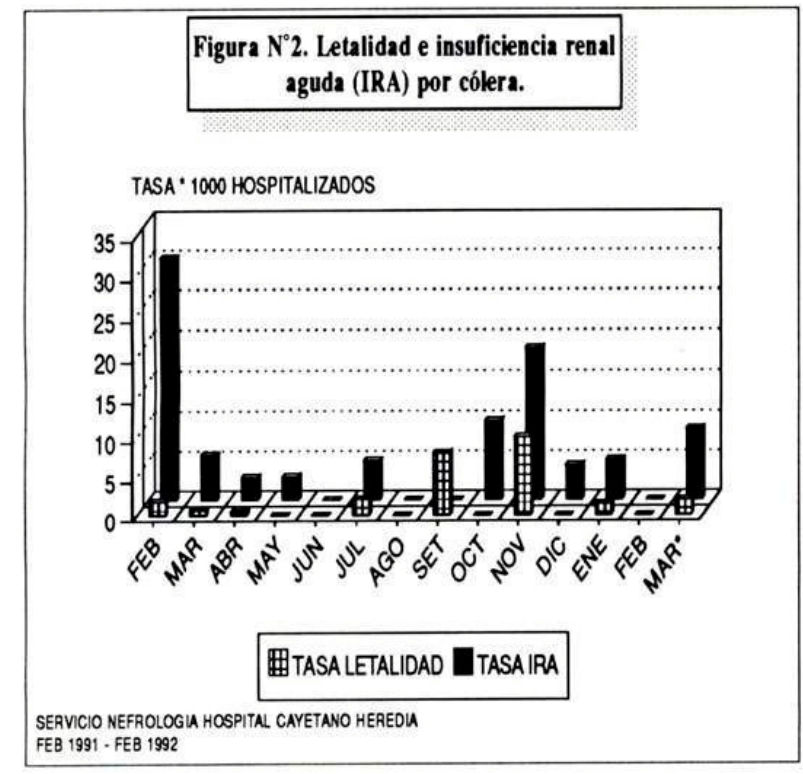

La morbilidad y la letalidad se vieron afectados en los períodos de escaso flujo de pacientes (junio agosto 1991). En este período, la eliminación del sistema operativo de rehidratación de pacientes con cólera, significó también ruptura de normas rutinarias en la atención de pacientes y ello se tradujo en un aumento de la letalidad y mortalidad (2).

Los recursos hospitalarios y la tecnología utilizada en pacientes complicados son otros puntos relevantes. Desde un inicio de la epidemia, dos hospitales de Lima fueron designados como hospitales de referencia nefrológica. Ambos por contar con unidades renales (servicios de diálisis, laboratorio nefrológico y unidades de cuidados intensivos).

Los resultados en la primera de la epidemia de Lima mostraron que los hospitales de referencia nefrológica (Cayetano Heredia y Arzobispo Loayza) presentaban las más bajas tasas de letalidad entre todos los hospitales de la ciudad (3). Un año fue muy diferente ( Figura No3). Mientras que la tasa disminuyó en casi todos los hospitales (probablemente por efecto de la mejoría de sus esquemas de rehidratación y por haber aprendido a derivar tempranamente a pacientes complicados a los hospitales de referencia), los hospitales de referencia nefrológica mostraron un aumento en sus tasas de letalidad. Esto puede explicarse por la calidad de pacientes referidos, de mucho mayor riesgo que el año anterior. En este punto, el tipo de tecnología usada resulta gravitante en la diferencia de los resultados. 


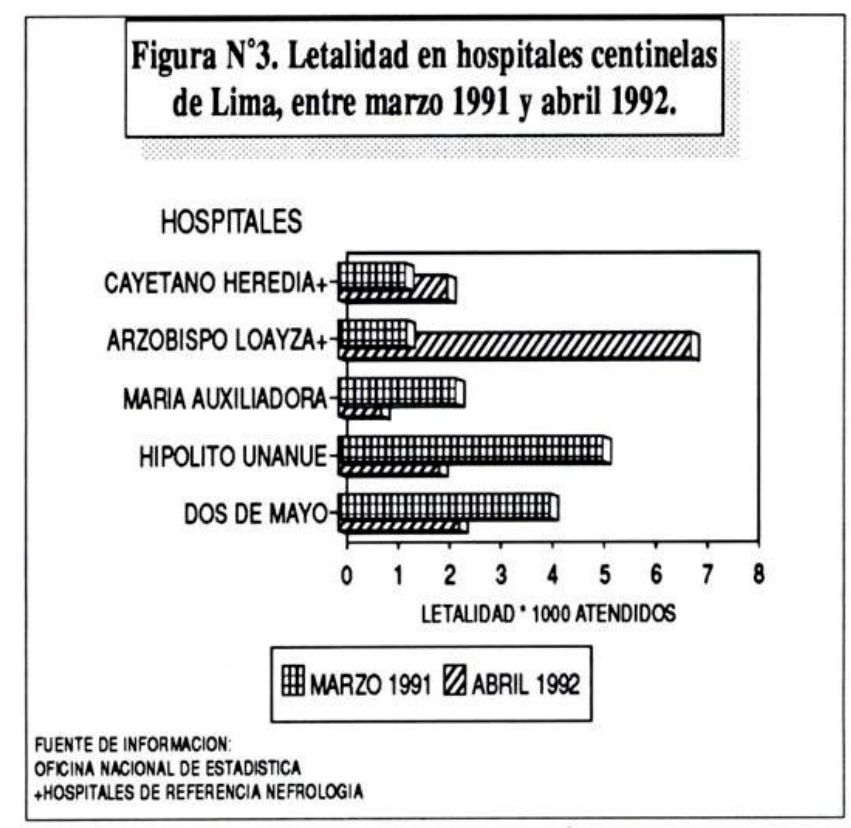

\section{TRATAMIENTO DE REHIDRATACION}

En relación al manejo individual del paciente, la terapia debe ser considerada en función de los siguientes parámetros:

1. Reconocimiento de las alteraciones fisiopatológicas del paciente deshidratado por cólera. Esta puede ser diferente según las características de la población afectada (edad y estado nutricional probablemente son las más relevantes).

2. Condiciones frecuentemente asociadas a riesgo de muerte. La experiencia peruana ha demostrado que cuando el paciente alcanza a ser atendido en un servicio de salud, el riesgo de muerte por hipovolémico es mínimo y está dado por la aparición de insuficiencia renal aguda $(2,4)$. En la terapia del paciente deshidratado, es importante realizar prevención secundaria de insuficiencia renal. Finalmente si esta ocurriera, el contar con una adecuada infraestructura y tecnología apropiada garantiza la mejor de la alternativa al paciente complicado.

3. El costo/beneficio del método de rehidratación sugerido. El tipo de tratamiento dependerá eminentemente de la institución donde se atienda al paciente. Si el servicio de salud se brinda en un hospital con gran demanda de pacientes, esquemas operativos sencillos, muy ágiles, y seguros deberán ser comparados en función de costos (5).

En las páginas anteriores, hemos apreciado que diversos trastornos pueden ocurrir en un paciente deshidratado por cólera. Sin embargo, en un primer momento, la hipovolemia y la hipoperfusión tisular son los aspectos críticos más importantes a solucionar.

Dado que la contracción de la volemia por cólera es isosmolal para la gran mayoría de los pacientes, la enérgica reposición inicial de la volemia con soluciones isosmolales es lo más importantes. Puesto que la corrección de las funciones vitales no garantiza necesariamente que la volemia se encuentre plenamente restablecida, es la recuperación de la diuresis lo que garantiza es restablecimiento de la volemia y una adecuada perfusión tisular. Por esta razón 
es muy importante no sólo restablece un adecuado nivel de la presión arterial, sino verificar su adecuado flujo urinario.

El detalle es más importante en ancianos, en quienes niveles de presión arterial considerados como normales pueden significar hipotensión arterial y confundir al terapeuta. El mantener mayor tiempo la hipoperfusión tisular, significa mayor isquemia renal y conlleva necesariamente mayor riesgo de necrosis tubular aguda. Son los ancianos, quienes partiendo de riñones menos tolerantes a la injuria por isquemia, desarrollan mayor incidencia de insuficiencia renal aguda.

El tipo de solución, necesariamente endovenosa, empleada en este período (que llamaremos de expansión rápida) puede ser de diferentes características, mas lo importante es que el tipo de solutos empleados contengan fuerza osmótica efectiva en el extracelular (cloruro de sodio al $0.9 \%$ o solución polielectrolítica Hartmann o equivalente). La experiencia en el Hospital Cayetano Heredia muestra que la expansión - hasta recuperar la diuresis - utilizando cloruro de sodio, permite corregir rápidamente el anion gap y mucho más lentamente la acidosis metabólica ( Figura $\mathrm{N}^{0} 4$ y figura $\mathrm{N}^{\mathrm{o}} 5$ ) sin presentar riesgo alguno sobre hipotéticas alteraciones del potasio o del sodio, e igualmente es eficaz en adultos jóvenes y ancianos $(6,7,8)$ ( Figura $\mathrm{N}^{\mathrm{o}} 6$ ). Otras soluciones isosmolales polielectrolíticas, principalmente la solución Hartmann, también son exitosas en la corrección de la hipovolemia, presentando la ventaja de la mejor corrección del anion gap y la acidosis metabólica sobre todo del enfermo severamente deshidratado ( Figura $\left.\mathrm{N}^{0} 7\right)(9,10)$. Nosotros creemos que la decisión del tipo de solución a emplear depende del costo y disponibilidad del insumo.
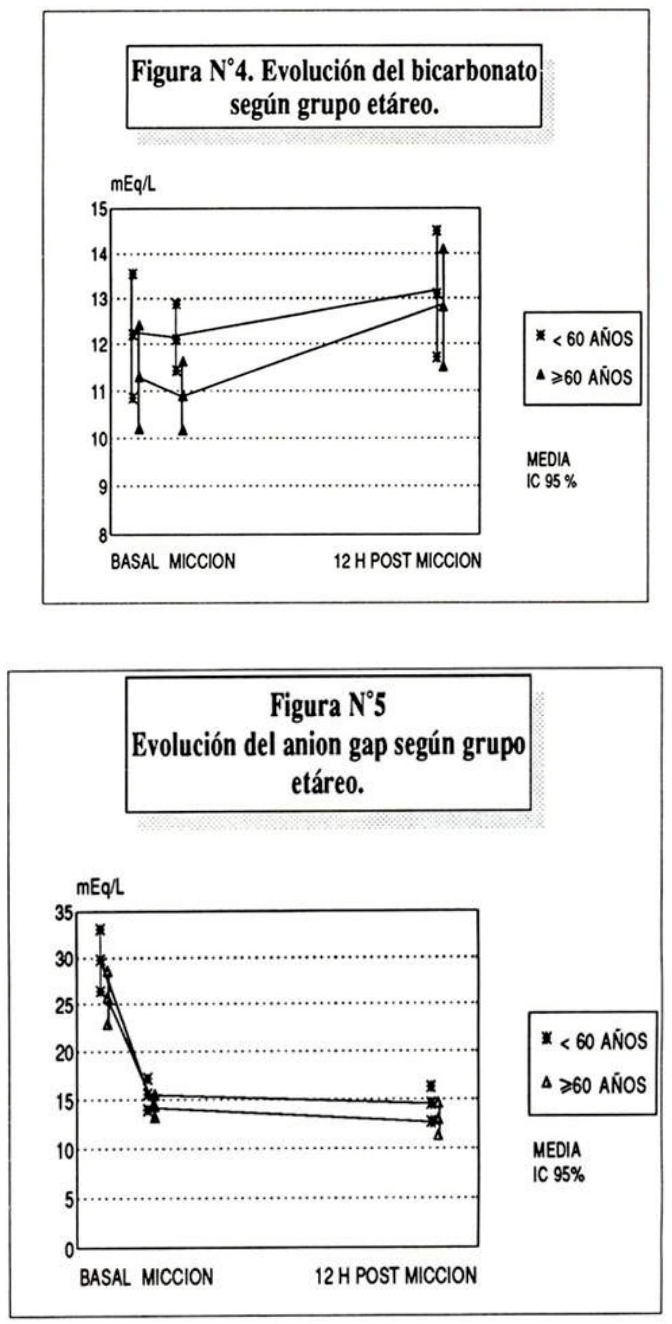

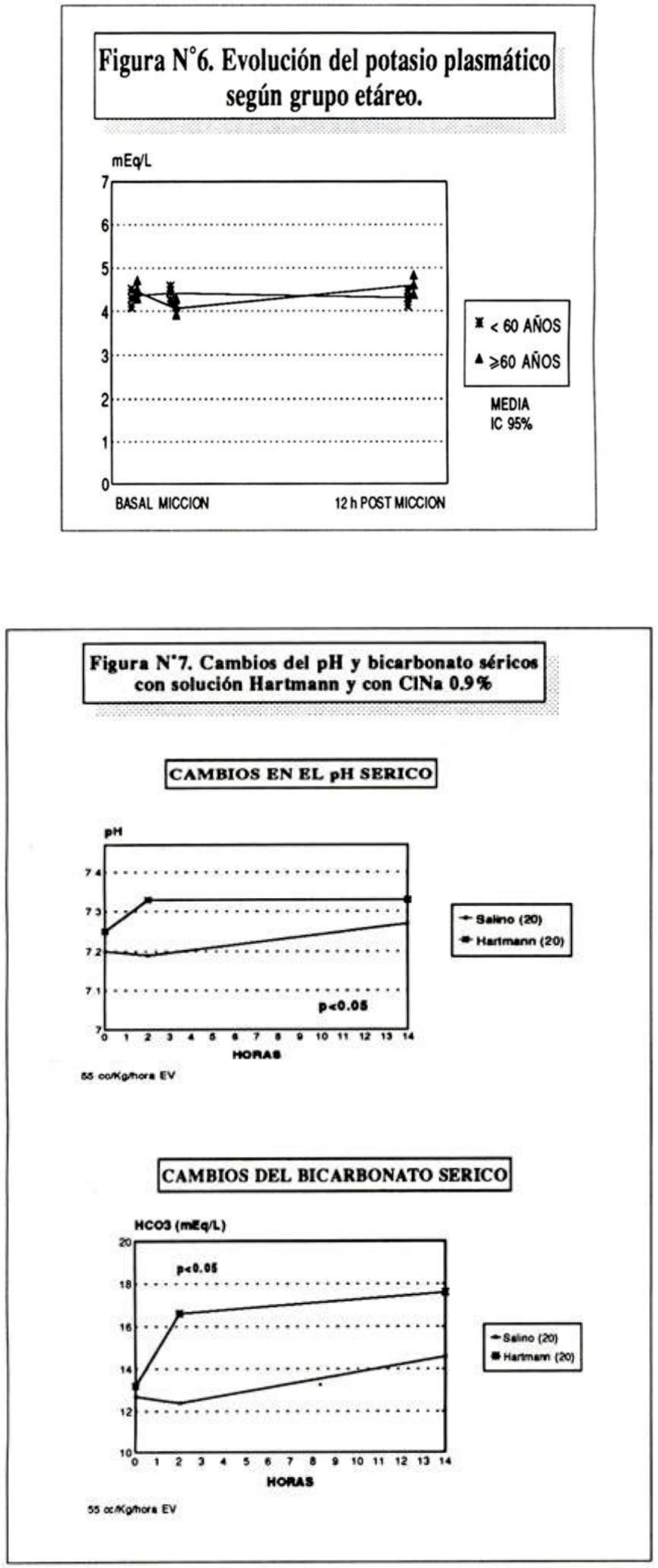

Al parecer, es la acidosis metabólica la responsable en gran medida de una serie de síntomas del paciente con cólera. El más importante de ellos es el vómito. Al recuperarse la diuresis, las náuseas y vómitos mejoran dramáticamente ( Figura $N^{\circ} 8$ ) y la hidratación oral puede 
utilizarse eficientemente. Desde este punto de vista, la solución de Hartmann ofrece la ventaja de permitir una más rápida transición a la hidratación oral. En este punto, es muy importante el inicio de soluciones polielectrolíticas en el mantenimiento de la hidratación del enfermo. Según las situaciones propias de cada institución y facilidades existentes, puede usarse solución polielectrolíticas endovenosa u oral de composición electrolítica similar a la de la diarrea, desde este momento hasta el fin de las mismas.

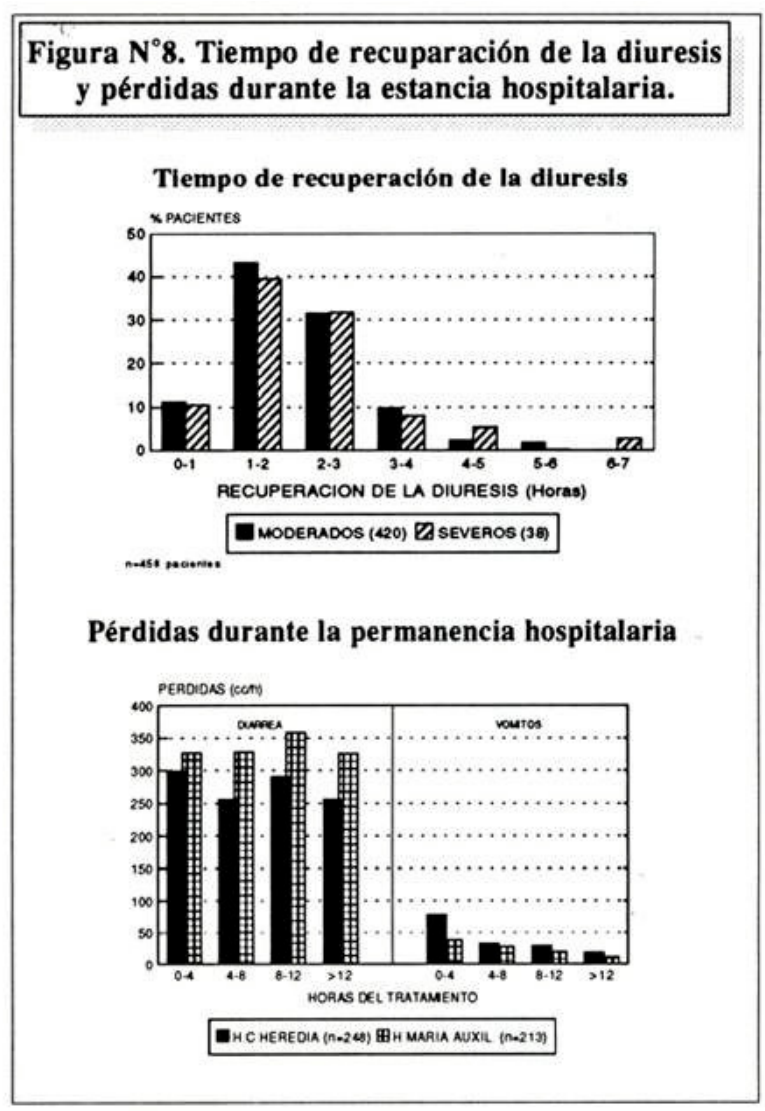

La corrección de los disturbios gasométricos y electrolíticos suelen ser posteriores a la mejoría clínica de los pacientes. Los deshidratados severos no muestran diferencias importantes en su evolución en relación a los deshidratados moderados. El riesgo de desarrollar IRA no está asociado a la gravedad de la deshidratación con la que el paciente es admitido, sino a la posibilidad de mantener exitosamente la hidratación con soluciones polielectrolíticas orales o endovenosas después que el paciente fue expandido y la diuresis restablecida (2).

La figura $\mathrm{N}^{\circ} 9$ presenta un resumen de las conductas terapéuticas empleadas en el Hospital Cayetano Heredia para el tratamiento de pacientes deshidratados por el cólera. 


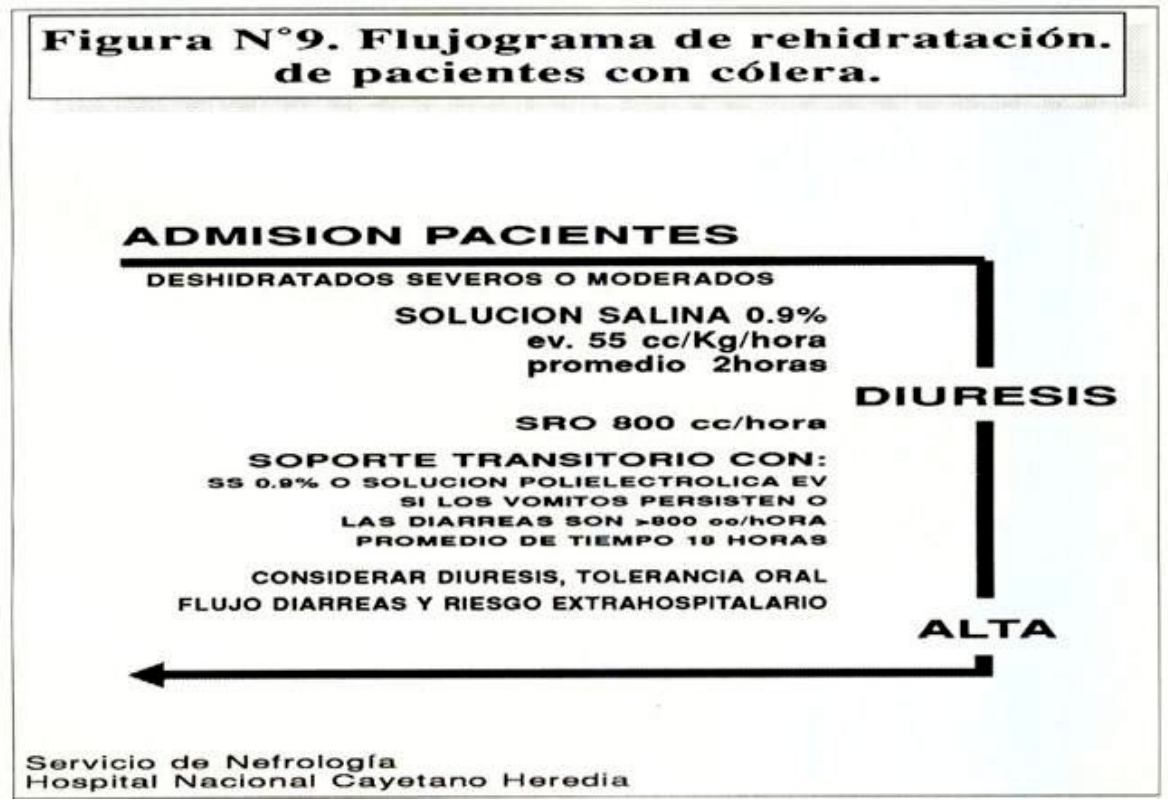

\section{REFERENCIAS BIBLIOGRAFICAS}

1. Mujica O, Seminario C, Tause L, et al. Investigación epidemiológica del Cólera en el Perú: Lecciones para un continente en riesgo. Rev Med Hered 1991: 2: 121-129.

2. Cieza J, Gutierrez R, Estremadoyro L, Miyahira J. Insuficiencia renal aguda secundaria a cólera. Aspectos epidemiológicos. Rev Med Hered 1992; 3: 140-147.

3. Cieza J, Gamarra G, Torres C. Letalidad y riesgo de insuficiencia renal por cólera en el Hospital Nacional Cayetano Heredia de Lima, Perú. Rev Med Hered 1991; 2: 75-78.

4. Miyahira J, Acosta R, Zurita S, Torres C. Insuficiencia renal aguda en el Hospital Nacional Cayetano Heredia durante la epidemia cólera. Rev Med Hered 1991; 2: 64-69.

5. Aliaga MP, Beraún S. Evaluación y comparación de dos esquemas de rehidratación en pacientes mayores de 14 años con enfermedad diarreica coleriforme en los hospitales Cayetano Heredia y María Auxiliadora en el verano de 1992. Tesis Lic Enfermería. Lima, Peru. Universidad Peruana Cayetano Heredia, 1993.

6. Cieza J, Gamarra G, Torres C, Miyahira J. Expansión endovenosa rápida con cloruro de sodio al $0.9 \%$, en pacientes adultos moderada y severamente deshidratados por cólera. Rev Med Hered 1991; 2: 57-63.

7. Cieza J, Gamarra G, Torres C, Gutierrez R, Estremadoyro L, Miyahira J. Evolución de los electrolitos séricos durante el tratamiento de rehidratación de pacientes con diarrea aguda coleriforme. Rev Med Hered 1993; 4: 3-11.

8. Sovero Y, Estremadoyro L, Cieza J. Alteraciones del medio interno durante la rehidratación de ancianos y adultos severamente deshidratados por diarrea coleriforme. Lima: I Congreso Regional Andino de Nefrología; 1994.

9. Carpenter CCJ. The erratic evolution of cholera theraphy: from folklore to science. Clin 
Theraphy 1990; 12 (suppl A): 22.

10. Hinostroza J, Sovero Y, Estremadoyro L, Cieza J. Efectos de la expansión endovenosa rápida con solución Hartmann y con cloruro de sodio al $0.9 \%$, sobre el medio interno de pacientes con deshidratación severa por diarrea aguda coleriforme. Rev Med Hered 1995; 6: 72-75.

\section{Correspondencia:}

Dr. Javier Cieza Cevallos

Servicio de Nefrología. Universidad Peruana Cayetano Heredia

Av. Honorio Delgado 430, San Martín de Porres

Lima, Perú. 